\title{
Inhibiting autophagy in dendritic cells
}

Down-
modulation of
autophagy in
[dendritic cells]
compromised
their ability
to present
autoantigen,
prime T cells
and induce
experimental
autoimmune
encephalo-
myelitis.

New findings have shed light on the molecular mechanisms by which regulatory $\mathrm{T}\left(\mathrm{T}_{\text {reg }}\right)$ cells suppress dendritic cells (DCs), thereby suppressing autoimmunity. " $\mathrm{T}_{\text {reg }}$ cells potently suppress autoimmune responses in vivo through inhibition of the autophagic machinery in dendritic cells in a cytotoxic T-lymphocyteassociated protein 4 (CTLA4)-dependent manner," states corresponding author Panayotis Verginis.

"Aberrant $\mathrm{T}_{\text {reg }}$ function has been closely linked to the development of autoimmune diseases and $\mathrm{T}_{\text {reg }}$ cell-based therapies hold great promise," explains Verginis. "The clinical implementation of these therapies however has been hampered by a lack of understanding on the mechanism of their action."
Verginis and colleagues sought to investigate the molecular targets underlying $\mathrm{T}_{\text {reg }}$ cell-mediated modulation of DC function, demonstrating in mice that autoantigen-specific $\mathrm{T}_{\text {reg }}$ cells inhibited induction of autophagy and autophagolysosome formation in DCs. Exposure of DCs to autoantigen-specific $T_{\text {reg }}$ cells downregulated the mechanistic target of rapamycin (mTOR) signalling pathway, a well-known regulatory pathway in autophagy.

Downmodulation of autophagy in

DCs compromised their ability to present autoantigen, prime T cells and induce experimental autoimmune encephalomyelitis. In the presence of a CTLA4blocking antibody, $\mathrm{T}_{\text {reg }}$ cells lost their ability to downregulate autophagy in DCs, leading Verginis and colleagues to investigate the

effects of abatacept (a CTLA4-Ig fusion protein) on murine bone-marrow-derived DCs. "Mechanistically, CTLA4 binding promoted activation of the mTOR pathway and Forkhead box protein O1 (FOXO1) nuclear exclusion in dendritic cells, leading to decreased transcription of the autophagy components," says Verginis. DCs derived from patients with rheumatoid arthritis being treated with abatacept also displayed diminished autophagy compared with those derived from patients with rheumatoid arthritis undergoing anti-TNF therapy.

"These findings are clinically relevant and of potential therapeutic use," remarks Verginis. Looking to the future, his group aim to focus on innovative methods to efficiently target autophagy in DCs. "Such an approach could pave the way for the development of new therapies not only in the field of autoimmunity but also in transplantation," Verginis concludes.

Jessica McHugh

ORIGINAL ARTICLE(S) Alissafi, T. et al. Tregs restrain dendritic cell autophagy to ameliorate autoimmunity. J. Clin. Invest. http://dx.doi. org/10.1172/ICl92079 (2017) 